Anglais de spécialité et milieux professionnels

\title{
Les milieux professionnels et leur régulation: diversité terminologique et complexité organisationnelle
}

Professions and their regulation: terminological diversity and organisational complexity

\section{Caroline Benedetto}

\section{OpenEdition \\ Journals}

Édition électronique

URL : http://journals.openedition.org/asp/4938

DOI : 10.4000/asp.4938

ISSN : 2108-6354

\section{Éditeur}

Groupe d'étude et de recherche en anglais de spécialité

\section{Édition imprimée}

Date de publication : 1 mars 2017

Pagination : 7-24

ISSN : 1246-8185

Référence électronique

Caroline Benedetto, « Les milieux professionnels et leur régulation : diversité terminologique et complexité organisationnelle », ASp [En ligne], 71 | 2017, mis en ligne le 01 mars 2018, consulté le 02 novembre 2020. URL : http://journals.openedition.org/asp/4938; DOI : https://doi.org/10.4000/asp. 4938

Ce document a été généré automatiquement le 2 novembre 2020.

Tous droits réservés 


\title{
Les milieux professionnels et leur régulation : diversité terminologique et complexité organisationnelle
}

\author{
Professions and their regulation: terminological diversity and organisational \\ complexity
}

Caroline Benedetto

\section{Introduction}

1 La régulation, qui vise l'organisation du fonctionnement des domaines spécialisés conçus comme systèmes complexes (Petit 2010 : \$22), occupe une place essentielle dans les milieux professionnels au Royaume-Uni. Elle désigne un vaste ensemble de pratiques qui permettent de veiller au respect des normes et de la réglementation, de contrôler l'acquisition des savoirs, d'élaborer des codes de conduite, ou encore de rappeler à l'ordre un praticien en lui demandant de respecter certaines obligations. Ces pratiques, comme le suggère la définition suivante proposée par un groupe de travail britannique chargé de réformer le système de régulation, influencent le comportement des professionnels :

regulation may widely be defined as any government measure or intervention that seeks to change the behaviour of individuals or groups. It can both give people rights [...] and restrict their behaviour. (BRTF $2003: 1$ )

2 Les pratiques de régulation incitent ou obligent effectivement les professionnels à se conformer à certaines exigences formulées par les institutions ou à certaines attentes exprimées par les usagers. Le discours de régulation, sur lequel nous centrons notre étude, influence lui aussi le comportement des praticiens car il énonce un ensemble de règles et de principes éthiques à respecter dans l'exercice d'une profession. 
3 Le corpus sur lequel se fonde cette étude réunit plusieurs textes de régulation publiés entre 1995 et 2016, tels que des rapports d'activité publiés par des associations d'usagers $^{1}$ et par des organismes publics chargés de contrôler les professions et les formations ${ }^{2}$, des codes de conduite ${ }^{3}$, des recommandations relatives aux bonnes pratiques $^{4}$ ou encore des guides destinés aux futurs membres des professions ${ }^{5}$.

4 Nous nous intéressons à trois milieux professionnels : ceux de la santé, du droit et de l'ingénierie. Ces milieux sont examinés selon une approche comparative visant à faire ressortir leurs convergences et leurs divergences en matière de pratiques et de discours de régulation. Notre travail se distingue d'autres études traditionnellement menées dans le champ des études anglophones en ce qu'il s'appuie sur l'observation des acteurs, des pratiques et des usages concrets rencontrés dans ces milieux, et sur les discours qui en découlent, plutôt que sur l'étude exclusive de la terminologie et des structures de la langue. Bien que notre démarche s'appuie sur l'analyse de certains termes clés, elle ne relève pas directement de l'analyse du discours ni de la terminologie : elle vise à faire ressortir, à travers le prisme de la régulation, certains traits stylistiques propres aux textes spécialisés afin d'apporter une meilleure compréhension des milieux qui les produisent.

Dans la première partie de cet article, nous soulignons que les milieux professionnels de la santé, du droit et de l'ingénierie sont emblématiques de la régulation qui s'exerce sur les domaines spécialisés. Puis, nous nous intéressons au discours de régulation produit par ces trois milieux professionnels: nous constatons qu'une diversité de termes et de sigles est employée pour désigner les acteurs de la régulation et ses dispositifs. Cette diversité reflète la complexité de l'organisation des milieux spécialisés.

\section{Santé, droit et ingénierie : des milieux représentatifs de la régulation qui s'exerce sur les domaines spécialisés}

6 Les milieux de la santé, du droit et de l'ingénierie sont, à plusieurs égards, représentatifs de la régulation qui s'exerce sur les domaines spécialisés.

7 En tant que «professions » au sens anglais du terme, les activités de travail accomplies dans ces trois milieux possèdent un statut particulier au sein de la société, comme le rappelle Everett Cherrington Hughes : " a profession is an occupation which has attained a special standing among occupations » (1958:157).

8 Le mot "profession" possède, comme le suggère cette définition, une acception traditionnelle particulière en anglais britannique: il désigne des activités de travail qualifiées, qui requièrent l'obtention d'une formation spécifique, longue et exigeante, généralement dispensée à l'université. Cette formation permet aux praticiens d'acquérir des savoirs d'expertise fondés sur un socle de connaissances abstraites, systématisées et codifiées, qui peuvent être difficilement saisies par un public de noninitiés.

9 L'exercice des professions ne peut se réduire à l'application de techniques apprises: il implique aussi, chez les praticiens, l'acquisition d'un ensemble de savoir-être et de comportements approuvés et partagés par les membres d'un même groupe 
professionnel. Les professions sont ainsi exercées en référence à un code d'éthique et de conduite dont les principes doivent être respectés par les praticiens.

Les professions de la santé, du droit et de l'ingénierie sont aussi représentatives de la régulation qui s'exerce sur les domaines professionnels car elles comportent un certain nombre de "barrières à l'entrée ». L'accès à ces professions repose effectivement sur le respect de certaines conditions, comme l'obtention d'une formation déterminée : il faut non seulement connaître la médecine pour être médecin, l'odontologie pour être dentiste, le droit pour être Solicitor ou Barrister, mais aussi avoir suivi une formation théorique et pratique dans l'une des institutions officiellement reconnues par les organismes responsables de la profession ${ }^{6}$.

11 L'accès aux professions du droit, de la santé et de l'ingénierie est, de plus, réservé aux membres qui possèdent un titre professionnel spécifique, protégé par la loi, comme ceux de Chartered Legal Executive ou de Chartered Engineer, agréés par Charte royale. Délivrés par les associations responsables de chaque spécialité (Professional Institutions), les titres professionnels constituent "un véritable filtre à l'entrée sur le marché du travail » (Le Bianic 2009: 488), en restreignant l'accès à une profession donnée aux seuls praticiens estimés compétents pour pouvoir l'exercer.

Outre la possession d'une formation déterminée et d'un titre spécifique, l'accès aux professions de la santé, du droit et de l'ingénierie requiert habituellement l'obtention d'un certain niveau d'expérience de travail (work experience), comme la réalisation de stages pratiques. Il arrive également que l'accès à ces professions soit conditionné à la réussite d'examens linguistiques. C'est le cas dans le domaine de la médecine (générale et spécialisée) où, depuis 2013, les candidats non anglophones doivent démontrer leurs compétences linguistiques en justifiant de l'obtention d'un score minimum à l' International English Language Testing System (IELTS) pour recevoir leur licence d'exercice (licence to practise) ${ }^{7}$.

Dans les milieux examinés, l'adhésion à une association professionnelle (professional membership) est aussi obligatoire ou fortement recommandée selon le domaine d'activité et/ou la spécialité envisagée ${ }^{8}$. Pour les praticiens, cette adhésion est garante d'une meilleure employabilité. Dans le domaine de l'ingénierie, par exemple, une étude menée sur un site généraliste de recherche d'offres d'emploi montre que plusieurs employeurs donnent la préférence aux candidats membres d'une association professionnelle reconnue. Les offres mentionnent, parmi leurs critères de sélection, " preferably Chartered status with a relevant professional body" ou encore "the minimum qualifications for this position are: be working toward Chartered Engineer status" (Indeed 2016) ${ }^{9}$. Ainsi, il n'est pas rare que certaines offres fassent référence à une association professionnelle en particulier, comme l'Institution of Chemical Engineers (IChemE) pour les ingénieurs chimistes ou la Chartered Building Services Engineering (CIBSE) pour les ingénieurs en équipement technique des bâtiments (Indeed 2016).

La mise en place de ces nombreuses «barrières à l'entrée » des milieux professionnels de la santé, du droit et de l'ingénierie est justifiée par le fait que les praticiens exercent fréquemment leurs activités de travail au contact du public. La régulation répond, par conséquent, à un souci de protection des usagers, comme en témoignent les propos suivants, formulés par le directeur de l'Engineering Council (EngC), et publiés sur le site d'une association d'ingénieurs : 
not every function needs to be protected. In the UK, the closer you are to working with the public instead of an informed client, the more likely your occupation is regulated. (IMechE 2015 : § 22)

15 L'introduction de la régulation dans certaines professions vise donc à garantir la protection des usagers ou celle de leur environnement, la qualité et la sécurité des produits ou des services proposés, ainsi que la compétence et le professionnalisme des praticiens.

16 Enfin, les professions de la santé, du droit et de l'ingénierie remplissent toutes une «fonction régulatrice» au sein de la société (Bourdoncle 1991: 86). Qu'il s'agisse de l'activité de soin pour les médecins, du règlement des différends pour les Barristers ou les Solicitors, ou de la mise en œuvre de services impliquant la résolution de problèmes techniques complexes pour les ingénieurs, les professions examinées présentent toutes une utilité fonctionnelle avérée. En répondant à des besoins indispensables de santé, de justice, de gestion et de résolution des conflits, elles participent «à la régulation et au contrôle menant au bon fonctionnement (smooth functioning) de la société » (Chapoulie 1973 : 92).

L'étude des textes de régulation en circulation dans les milieux de la santé, du droit et de l'ingénierie met en évidence une grande diversité terminologique dans la désignation des acteurs de la régulation. La diversité des termes employés montre que l'organisation des domaines spécialisés s'est complexifiée depuis le début des années 1990.

\section{Diversité terminologique}

L'analyse de la terminologie, comme le rappelle Catherine Resche, apporte de précieux enseignements quant à la construction et à l'évolution des domaines spécialisés (2013). L'étude des termes clés et de leurs variations permet de révéler non seulement le caractère spécialisé des domaines, mais aussi l'influence du contexte social sur ces derniers (ibidem). L'auteur prône ainsi un travail sur le discours réalisé selon une perspective diachronique :

a dynamic approach that takes into consideration historical, textual and situational contexts in order to observe and analyse how concepts and terms evolve, what influences have been brought to bear on the evolution of a given domain, how discourse is adapted to targeted audiences, and how it can be seen as a mirror as well as a motor of change. (Resche, $2015: 215$ )

19 Une telle approche, qui envisage les domaines spécialisés comme le produit de cultures, de normes et de conventions spécifiques (Resche 2013), est celle que nous retenons dans le cadre de notre analyse des pratiques de régulation. Nous envisageons ainsi les paradigmes de désignation des acteurs et des dispositifs de contrôle comme une voie d'accès vers la conception de la régulation qui structure les activités des milieux professionnels de la santé, du droit et de l'ingénierie.

20 Le discours de régulation des milieux de la santé, du droit et de l'ingénierie est marqué par une grande diversité de termes employés pour désigner les acteurs et les dispositifs de régulation. On examinera successivement les termes clés ainsi que les sigles et acronymes qui y font référence en vue de faire ressortir certaines spécificités des milieux professionnels examinés. Notre intention n'est pas de proposer un inventaire exhaustif des acteurs ou des dispositifs de régulation existants, ni des politiques dans 
lesquelles ils s'insèrent, mais de mettre en évidence, à travers quelques exemples tirés du discours de régulation, les changements récents qui caractérisent les milieux de la santé, du droit et de l'ingénierie. L'étude des termes clés et des sigles en présence dans le discours de régulation doit nous permettre de mieux appréhender la réalité de terrain et l'identité de ces milieux.

21 Les termes clés et les sigles que nous avons relevés au cours de nos lectures appartiennent tous aux domaines de la santé, du droit et de l'ingénierie. Toutefois, comme nous le montrons dans la section [2.1.2.], certains des termes employés, en particulier ceux qui désignent les dispositifs de régulation, ont été empruntés à d'autres domaines d'application comme le domaine militaire, celui du management des organisations ou celui du marketing.

\subsection{Termes clés}

\subsubsection{Acteurs de régulation}

L'étude des termes clés présents dans le discours de régulation, en particulier dans les rapports d'information destinés au grand public, montre qu'une terminologie hétérogène est employée, tant pour désigner les acteurs collectifs de la régulation que les individus régulés.

Les organismes de régulation répondent aux appellations très génériques de " regulators ", « regulatory bodies ", " approved regulators ", " front-line regulators ", "professional bodies», " authorised bodies» ou encore "recognised supervisory bodies». Les praticiens sont quant à eux nommés "the regulated», "regulated entities ", « regulated individuals ", « regulated persons », « authorised persons » voire « regulated and unregulated providers ».

Quand on examine les appellations spécifiques données aux organismes de régulation, on rencontre, à nouveau, une grande diversité. Dans le milieu du droit, Institute, Authority, Board et Council sont les principaux termes employés (Chartered Institute for Legal Executives, Solicitors Regulation Authority, Bar Standards Board, Bar Council). Dans le milieu de la santé, Council est le terme le plus répandu (Nursing and Midwifery Council, General Dental Council, General Osteopathic Council) ; on y rencontre aussi les termes " Colleges" ou "Royal Colleges" qui font référence aux associations professionnelles. Enfin, dans le milieu de l'ingénierie, on retrouve le terme "Council» qui désigne l'organisme de régulation de la profession; on relève également les termes Institute et Institution qui désignent la plupart des associations professionnelles d'ingénieurs (Chartered Institution of Building Services Engineers, Chartered Institution of Highways and Transportation, Institution of Civil Engineers, Institution of Chemical Engineers).

On observe également une grande variabilité en ce qui concerne le statut des organismes de régulation: certains d'entre eux sont des agences du gouvernement (executive agencies) comme la Medicines and Healthcare products Regulatory Agency, d'autres sont des "Non-departmental public bodies $»^{10}$ comme la Care Quality Commission ou le Legal Services Board. On trouve enfin des "registered charities" (organismes à but non lucratif) comme l'Engineering Council ou le General Medical Council.

L'emploi des différents termes désignant les régulateurs peut semer la confusion dans l'esprit des non-initiés. Car si le public a une idée générale de ce que signifie "régulation », il ne connaît pas toujours précisément les compétences spécifiques de 
chaque régulateur et ne sait pas nécessairement quels sont les membres de ces structures. Plusieurs organismes de régulation s'efforcent ainsi d'expliciter leur propre rôle ou celui d'autres instances du domaine par l'intermédiaire de leur site internet ${ }^{11}$. La diffusion d'informations concernant les régulateurs doit garantir une meilleure lisibilité du système pour le grand public.

La diversité des termes qui font référence aux organismes de régulation et à leurs statuts nécessite d'être mise en lien avec l'histoire des domaines spécialisés, et plus particulièrement avec les origines de leur construction. Dans le domaine du droit, par exemple, les Inns of Court, dont la création remonte au XIII siècle, sont des instituts où se déroulaient les enseignements théoriques et pratiques des Barristers. Leurs membres responsables contrôlaient l'ensemble des activités mises en œuvre par les avocats et se chargeaient de faire respecter la déontologie de la profession. Dans le domaine de la santé, certains Royal Colleges, instituts traditionnellement responsables de la formation, datent du XVI ${ }^{e}$ siècle. C'est le cas, notamment, du Royal College of Physicians of London, fondé en 1518, qui accordait des licences aux médecins qualifiés et imposait des sanctions aux praticiens soupçonnés de mauvaises pratiques. Aujourd'hui, ces associations professionnelles semblent moins impliquées que par le passé dans l'élaboration et l'application des dispositifs de régulation. Elles s'attachent surtout à représenter et à défendre les intérêts d'une profession donnée, ainsi qu'à promouvoir, dans le cadre de la formation et de l'exercice professionnel, la diffusion des connaissances scientifiques et des bonnes pratiques.

La diversité terminologique constitue sans doute aussi une sorte de tropisme de l'identité britannique dans la mesure où, au Royaume-Uni, les institutions disposent traditionnellement d'une grande marge d'autonomie. Cette autonomie s'exerce non seulement aux niveaux universitaire, financier et du recrutement, mais aussi au niveau organisationnel, pour ce qui est du choix de dénomination de chaque structure. Les établissements d'enseignement supérieur (higher education institutions, HEIs) du Royaume-Uni, par exemple, forment un groupe très hétérogène, aux appellations diverses, comme le rappelle Marie-Agnès Détourbe : « ancient universities » comme celles d'Oxford, de Cambridge, de St Andrews ou de Londres, «civic universities » fondées à la fin du XIXe et au début du XXe siècle, " colleges of advanced technologies ", établissements ayant acquis le statut d'université après la publication de la loi de 1992, university colleges, colleges of higher education... (2011: 62-64). Ces établissements sont tous des " organisations autonomes et légalement indépendantes qui jouissent du statut de charities " (ibidem). Notons enfin que ces divers établissements bénéficient d'une marge d'autonomie dans la désignation des diplômes ${ }^{12}$ qu'ils délivrent aux membres des professions.

29 Les modes de désignation des acteurs de la régulation se caractérisent non seulement par une grande hétérogénéité, mais connaissent aussi un important processus de création lexicale. De nombreux termes sont ainsi apparus au cours des années 2000 pour désigner les organismes chargés de surveiller l'action des régulateurs ainsi que les associations qui défendent les intérêts des usagers. Les appellations données à ces structures, comme Healthwatch, Energywatch, Monitor, Inspectorate ou encore Watchdog, montrent que les praticiens sont désormais placés sous étroite surveillance. L'apparition de ces termes montre que le système actuel de régulation évolue vers une meilleure prise en compte des besoins des usagers en instaurant de nouveaux organismes qui leur sont dédiés. Mais comme le signalent certains auteurs (Kober- 
Smith \& Feroni, 2005 : 469-494 ; Kober-Smith, 2010 : 69-86 ; Maroy, 2007 : 87-98), l'intervention de ces nouveaux acteurs risque, à terme, d'entraîner une diminution de l'autorité et de la crédibilité des professionnels, qui font l'objet de contrôles plus nombreux dans le cadre de leurs activités.

\subsubsection{Dispositifs de régulation}

L'étude des modes de désignation des dispositifs de régulation montre que plusieurs termes ont été empruntés à d'autres domaines d'application que ceux de la santé, du droit ou de l'ingénierie. Ces emprunts montrent que la régulation s'est renforcée et qu'elle s'accompagne de contraintes plus nombreuses pour les professionnels des milieux examinés.

31 À titre d'exemple, on relève, dans les rapports publiés par les organismes de régulation en particulier, l'emploi de l'expression «command and control». Dans son acception première qui provient du domaine militaire, cette expression désigne à la fois «the functional exercise of authority, based upon knowledge, to attain an objective or goal» (command) et «the process of verifying and correcting activity such that the objective or goal of command is accomplished» (control), d'après la définition proposée par une association américaine spécialisée dans les questions de défense et de sécurité (Federation of American Scientists, FAS : 2016 : §4).

On retrouve l'expression «command and control» non seulement dans le domaine militaire mais aussi dans celui du management des organisations. Cette expression renvoie, en effet, au "modèle vertical et hiérarchique de la réglementation centralisée " (Raffenne 2010: \$10) de certains secteurs d'activité comme celui de la santé. Le modèle de "command and control » a toutefois été remplacé, au cours des années 2000, par un modèle appelé "empowerment », dont le discours et les dispositifs sont centrés « sur la participation et l'implication des usagers dans la gestion de leurs services"(ibidem). Ces deux modèles de régulation peuvent revêtir un caractère contraignant pour les professionnels dans la mesure où ils sont mis en œuvre par des acteurs externes aux professions, et où ils prévoient des dispositifs de sanctions en cas de non-respect des règles en vigueur.

Un autre terme que l'on retrouve fréquemment dans le discours de régulation est celui d'« accountability». Ce terme, qui relève du domaine de la comptabilité et de la gestion financière, désignait étymologiquement une obligation de reddition de comptes (imposée à une entreprise ou à une organisation), comme le rappellent Mark Bovens et al. :

The idea of accountability is historically rooted in the practice of book-keeping and in the discipline of accounting [...]. Accounting always has a dual meaning: it is about listing and counting important 'things' - possessions, debts, agreements, promises - and about providing an account concerning this count. Thus it implies telling a story, based on some obligation and with some consequence in view. (2014:2)

Depuis le début des années 2000, le terme d'« accountability» apparaît désormais dans des domaines professionnels variés, où il fait référence à l'obligation faite à un praticien de s'acquitter d'une tâche et de répondre de son exécution, à son supérieur ou à l'autorité compétente (" the obligation to provide an account to, usually, a superior or at least someone with a legitimate stake", selon Bovens et al. 2014: 3). Ainsi, la traduction de ce terme par «responsabilité professionnelle dans un contexte 
hiérarchique " nous paraît correspondre à la réalité de terrain des praticiens des domaines spécialisés.

la mesure où «l'insistance sur le côté money, en avoir pour son argent, s'estompe en partie en faveur d'une affirmation de la valeur spécifique des services publics" (Jeannot 1998 : 32). C'est d'ailleurs ce que confirment les propos suivants, formulés par le directeur d'une association d'usagers (Consumer Council for Water) :

value for money is not simply about lower bills - it's about water companies showing their customers that they are delivering reliable services and making the improvements customers expect. (2015 : \$5)

ession "value for money » exprime donc moins l'intérêt dédié à l'amélioration du rendement que celui porté à la satisfaction des usagers, dans le cadre de l'approche managériale des services publics au Royaume-Uni.

L'importance accordée à la satisfaction des usagers sert à introduire des outils d'évaluation, telles que des enquêtes de satisfaction, visant à mesure l'efficacité et l'efficience des professionnels, qui sont désormais tenus d'«apporter des preuves tangibles et mesurables de la valeur de leur investissement » (Kober-Smith, 2010: §7). 
Elle permet en outre d'introduire des services de réclamation et de compensation destinés aux usagers, qui peuvent désormais se plaindre, s'ils l'estiment nécessaire, de la qualité des services reçus. La prise en compte de la satisfaction des usagers peut ainsi représenter une mesure contraignante pour les professionnels car des sanctions disciplinaires sont prévues en cas de manquement au respect d'une obligation.

Dans le discours des milieux de la santé, du droit et de l'ingénierie, l'apparition de termes empruntés au management des organisations ou au marketing semble indiquer un changement de modèle de régulation. Désormais, la régulation s'exprime par des dispositifs externes aux professions, comme le montrent l'emploi des termes de " command and control» et d' 'empowerment » des usagers, et le remplacement du premier terme par le second.

42 Le passage d'une régulation mise en œuvre par les pouvoirs publics à une régulation émanant des usagers modifie inévitablement le rapport entre les acteurs des milieux professionnels, ainsi que leurs rôles respectifs. L'emploi du terme "value for money » est particulièrement révélateur de ces changements : il signale une modification du statut des professionnels, qui semble être passé de celui d'expert à celui de simple prestataire de service (service provider).

Certains des termes examinés plus haut mettent aussi en évidence une intensification de la fonction de régulation. Dans plusieurs milieux professionnels, comme celui de la santé, la notion de benchmarking montre que l'exigence de performance est devenue un enjeu majeur. L'emploi de ce terme, orienté vers la recherche des meilleures pratiques en vue de répondre aux attentes des patients, permet d'initier des changements culturels et organisationnels importants.

Enfin, l'introduction de la notion d'accountability dans le discours de régulation impose de nouvelles contraintes aux professionnels, qui sont désormais tenus de « rendre des comptes » à l'ensemble de la société. On peut ainsi se demander si l'apparition de cette notion ne risque pas de nuire à leur crédibilité auprès du public et de réduire leur autonomie. Si la promotion de la transparence est envisagée par les pouvoirs publics comme une réponse à la crise de confiance et de légitimité qui affecte la réputation des professions, elle témoigne aussi d'une certaine défiance vis-à-vis de la capacité des praticiens à remplir correctement leurs missions. Christian Maroy évoque à cet égard une " perte de confiance des autorités [...] dans la capacité autorégulatrice des milieux professionnels [...], dans leur conscience éthique et dans leur capacité technique à développer et à améliorer leurs pratiques et performances de façon autonome » (2007 : \$33).

45 Tout comme les termes clés qui sont employés pour désigner les acteurs de la régulation et ses dispositifs, les sigles et les acronymes manifestent une grande hétérogénéité.

\subsection{Sigles et acronymes}

Le discours de régulation est marqué par un important phénomène de siglaison. La présence de sigles et d'acronymes apporte un éclairage précieux sur les réalités de terrain rencontrées par les praticiens des milieux de la santé, du droit et de l'ingénierie: elle montre non seulement le caractère spécialisé de ces milieux, mais révèle aussi la multiplicité des acteurs et des dispositifs de régulation qui les caractérise. 
47 Le choix de recourir aux sigles et aux acronymes répond moins à un besoin «d'économie graphique» (Dufour 2011: 75) qu'à la volonté de sélectionner «une potentialité signifiante du sigle qui convient mieux à la réalité désignée et au contexte du propos tenu » (ibidem : 81). Quand on examine les glossaires qui accompagnent les rapports sur la régulation, on voit que ces formes abrégées confèrent une dénomination aux acteurs des contrôles (CLC, Council for Licensed Conveyancers; GOsC, General Ostheopatic Council) ainsi qu'aux associations chargées de diffuser les standards de chaque profession (RCGP, Royal College of General Practitioners ; IChemE, Institution of Chemical Engineers). D'autres sigles servent à désigner les dispositifs de régulation, comme la réglementation (LSA, Legal Services Act 2007) ou les processus d'assurance qualité (QASA, Quality Assurance Scheme for Advocates). Enfin, les sigles renvoient aux formations agréées (LPC, Legal Practice Course), aux titres professionnels (EngTech, Engineering Technician; IEng, Incorporated Engineer ; CEng, Chartered Engineer), ou aux diplômes agréés par les organismes de régulation (QLD, Qualifying law degree; MEng, Master of Engineering, $\mathrm{MBChB}$, Bachelor of Medicine and Bachelor of Surgery $\left.{ }^{14}\right)$. En tant que marqueurs d'identification, les sigles mettent en évidence la multiplicité des acteurs et des dispositifs de régulation qui caractérisent les milieux professionnels.

Sigles et acronymes se sont multipliés à la suite de la séparation, au début des années 2000, des fonctions de contrôle et de représentation des intérêts des praticiens. Si cette mesure vise à garantir l'impartialité de l'action des organismes de régulation, elle a aussi contribué à créer " a whole new raft of organisations and acronyms, so the overall size of the maze is not that much smaller", comme le note un Solicitor (Rose 2011 : §3). La prolifération des acronymes servant la désignation de ces structures a introduit une opacité terminologique élevée au sein des domaines spécialisés. L’identification des syntagmes qui correspondent aux sigles et aux acronymes peut effectivement être difficile pour le grand public, comme le signale Françoise Dufour :

le déploiement à partir de sigles bien connus demande un effort aux sujets sollicités, fait appel à l'improvisation et il en résulte souvent une certaine imprécision. (2011: 75)

L'étude des sigles et des acronymes montre que ces formes ne sont pas immuables, et que leur emploi et leur développement connaissent « des variations synchroniques et diachroniques ", comme le signale Jacqueline Percebois (2001: 636). On peut appréhender, à travers la modification de ces formes abrégées, les nombreux changements qui marquent le paysage de la régulation au Royaume-Uni : fusion, création ou suppression d'organismes de régulation, élaboration de nouveaux dispositifs de certification ou d'assurance qualité, mise en œuvre d'une nouvelle réglementation, actualisation des normes professionnelles et des référentiels de compétences. L'obsolescence des sigles et des acronymes, tout particulièrement, conduit à s'interroger sur les causes de la disparition ou du changement de nom des régulateurs. Il nous semble que certains organismes sont supprimés ou qu'ils fusionnent avec d'autres structures pour éviter une duplication des contrôles et pour proposer aux usagers un point d'accès unique en cas de recours ou de plainte. D'autres fusionnent afin de réunir un nombre élevé de membres relevant des mêmes champs d'application, et ainsi de donner davantage de poids et de crédibilité à leur structure ${ }^{15}$. En définitive, la modification constante du paysage de la régulation ainsi que l'absence de pérennité des organismes chargés des contrôles soulèvent certaines interrogations quant à l'efficacité et à l'utilité du rôle des régulateurs. 
Le discours de régulation se caractérise ainsi par une grande diversité terminologique, comme en témoignent la diversité des appellations et des statuts conférés aux régulateurs, les emprunts à d'autres domaines d'application, et la prolifération des sigles et acronymes qui vient d'être évoquée. Cette diversité terminologique est le reflet d'une organisation de plus en plus complexe, dont nous examinons les principales caractéristiques dans les sections suivantes.

\section{Complexité organisationnelle}

51 L'étude de l'organisation des milieux professionnels de la santé, du droit et de l'ingénierie montre que la régulation est l'œuvre d'une pluralité d'acteurs :

one speaks of a 'regulatory landscape' involving not only actors such as state institutions (e.g. ministries, departments, agencies, supra-national bodies such as the EU, international bodies such as the WTO) and non-state institutions (e.g. firms, committees, associations, and networks) but also economic (e.g. the market) and social conditions (e.g. norms, cognitive frames, technologies). (Spada 2009 : 13)

Ces acteurs, aux intérêts et aux objectifs distincts, conditionnent l'organisation, l'élaboration et la mise en œuvre des contrôles qui sous-tendent les activités des domaines spécialisés.

Outre-Manche, l'intervention directe de l'État dans la régulation des domaines spécialisés n'est pas une règle absolue. Tandis que la régulation demeure fortement centralisée en France, le Royaume-Uni a opté pour un système différent, au sein duquel les pouvoirs de contrôle sont de plus en plus souvent confiés à des structures indépendantes qui agissent en tant qu'intermédiaires entre l'État et les professions. Des organismes publics de régulation, comme l'Engineering Council (EngC) pour les ingénieurs ou le General Medical Council (GMC) pour les médecins, interviennent ainsi régulièrement dans l'élaboration et l'application des contrôles. Ils mettent en place des normes de standardisation relatives à l'ensemble d'une profession, définissent les compétences à atteindre, délivrent des titres et des certifications aux praticiens, édictent des règles ou des recommandations de bonnes pratiques, prévoient des sanctions en cas de manquement au respect des codes de conduite et ont le pouvoir de les appliquer.

Les organismes de régulation au Royaume-Uni sont très nombreux et il peut même en exister plusieurs pour un seul et même domaine spécialisé. Les domaines de la santé ou $\mathrm{du}$ droit sont ainsi régulés par neuf organismes ${ }^{16}$, chacun d'entre eux étant responsable d'une ou de plusieurs spécialités. Dans le domaine de l'ingénierie, en revanche, la fonction de régulation est assurée par un seul et même organisme, l'Engineering Council, tandis que les associations professionnelles (Professional Institutions), comme la Royal Academy of Engineering, se chargent de représenter les intérêts des ingénieurs et ne jouent qu'un rôle consultatif dans l'élaboration des dispositifs de régulation.

Outre le nombre important d'acteurs chargés des contrôles, on assiste à une hiérarchisation des différents organismes de régulation. C'est le cas, par exemple, dans le milieu de la santé, où un organisme appelé « Professional Standards Authority for Health and Social Care" joue le rôle de "meta regulator» ou d' "oversight regulator» en supervisant l'action des neuf organismes professionnels de régulation. Il en est de même dans le milieu du droit, où le Legal Service Board contrôle les activités des neuf organismes de régulation responsables des services juridiques. Le milieu de l'ingénierie 
présente lui aussi une hiérarchisation de ses organismes de régulation: l'Engineering Council se charge de vérifier que les associations d'ingénieurs ont bien contrôlé les critères requis avant d'inscrire les professionnels à son registre (registration).

La hiérarchisation des organismes chargés des contrôles peut rendre difficile la compréhension du système de régulation par les professionnels, qui ne savent pas toujours à quel organisme s'adresser. Dans leur discours, l'emploi d'expressions telles que "plethora », « myriad » ou " raft of regulators » souligne la complexité du système de régulation, du fait de la multitude d'acteurs intervenant dans l'élaboration et la mise en œuvre des contrôles.

Il arrive également que certains services soient doublement régulés (doubly regulated). C'est, par exemple, le cas dans le domaine du droit, où les avocats en cession de droits immobiliers (Licensed Conveyancers) sont tenus de respecter à la fois les critères requis par leur organisme de régulation, le Council for Licensed Conveyancers et les critères énoncés par la Law Society dans le cadre de sa démarche qualité (Rose 2013: \$1). Ce phénomène de « double régulation » ajoute à la lourdeur administrative à laquelle sont confrontés les professionnels, comme en témoigne, dans leur discours, l'emploi récurrent des expressions «regulatory maze» et "regulatory burden». La double régulation peut aussi entraîner des incompatibilités entre les règles et poser des problèmes d'interaction entre les différents acteurs des milieux professionnels.

\section{Conclusion}

58 L'étude des textes de régulation produits par les milieux de la santé, du droit et de l'ingénierie met en évidence plusieurs phénomènes linguistiques: diversité terminologique, création lexicale, emprunts à d'autres domaines professionnels, foisonnement des sigles et des acronymes, mais aussi fusion ou disparition de ces formes abrégées et des dénominations auxquelles elles renvoient. Ces phénomènes linguistiques, qui servent tous à nommer les régulateurs et les dispositifs de contrôle, montrent que l'organisation de la régulation des milieux professionnels s'est complexifiée depuis les années 1990: elle se caractérise par un accroissement du nombre de régulateurs et de contraintes qui pèsent sur les professionnels, qui sont de plus en plus tenus de rendre des comptes aux pouvoirs publics et à la société.

59 La variabilité des termes et des sigles employés pour désigner les acteurs et les dispositifs de régulation rend difficile la compréhension du système par les professionnels comme par les usagers. La représentation du système de régulation à l'œuvre dans le discours soulève donc un paradoxe : la régulation, qui a pour principal enjeu l'organisation du fonctionnement des domaines spécialisés, ne contribue-t-elle pas à en donner une image de complexité excessive, tant auprès du public qu'auprès des professionnels, et ainsi à rendre ardues son application et son acceptation par l'ensemble de la société ?

60 Plusieurs stratégies de clarification sont mises en œuvre, depuis le début des années 2000, pour remédier à l'opacité terminologique et à la complexité organisationnelle qui caractérisent la régulation des domaines spécialisés. Comme en témoigne la publication massive de rapports d'activité, de normes de bonnes pratiques et de référentiels de compétences, les régulateurs s'efforcent de privilégier la transparence de leur action 
afin de la rendre la plus acceptable possible par les professionnels comme par les usagers.

61 Sur le plan linguistique, la volonté des institutions de simplifier le langage de la régulation s'exprime tout particulièrement par le recours à un anglais clair et compréhensible dépourvu de jargon et d'acronymes (Plain English) ${ }^{17}$, par la publication de glossaires accompagnant les rapports destinés au grand public, comme le «NHS jargon and acronym buster ", ou par la rédaction de guides explicatifs au contenu allégé et vulgarisé comme le guide des avocats appelé "Shorter, sharper, clearer Handbook» publié par la Solicitors Regulation Authority (SRA 2016).

Les efforts réalisés en matière de communication doivent permettre une meilleure compréhension entre acteurs, de façon à diminuer le risque de recevoir des plaintes émises à l'encontre des praticiens et, à terme, à accroître la satisfaction des « usagers consommateurs » vis-à-vis des services reçus.

\section{BIBLIOGRAPHIE}

\section{Sources primaires}

Bar Council. 2013. Your Career as a Barrister. Londres. [Bar Council]

Better Regulation Task Force. 2003. Principles of Good Regulation. Londres. [BRTF]

Consumer Council for Water. 2015. « More customers satisfied with value for money of water and sewerage services ». Birmingham. [CCWater]

Department for Constitutional Affairs. 2005. The Future of Legal Services: Putting Consumers First. Londres. [DCA]

Federation of American Scientists. « Fundamentals of Naval Weapons Systems: Chapter 20 : Command, Control, and Communication ». Washington D.C. [FAS]

General Medical Council. 2009. Tomorrow's Doctors, Londres : GMC.

<www.gmc-uk.org/education/undergraduate/tomorrows_doctors_2009.asp>, consulté le 10/07/2016. [GMC]

General Medical Council. 2013. The State of Medical Education and Practice in the UK. Londres. [GMC]

General Medical Council. 1995-2013. Good Medical Practice, Londres : GMC. <www.gmc-uk.org/ guidance/good_medical_practice.asp>, consulté le 15/07/2016. [GMC]

General Medical Council. 2016. «Bodies awarding UK Medical Degrees », Londres : GMC. $<$ www.gmc-uk.org/education/undergraduate/awarding_bodies.asp>, consulté le 11/07/2016. [GMC]

Health and Care Professions Council. 2011. Professionalism in Healthcare Professionals. Londres : HCPC. [HCPC]

Institution of Civil Engineers. 2014. ICE Code of Professional Conduct. Londres : ICE. [ICE] 
Institution of Mechanical Engineers. 2015. « Excusez-moi, êtes-vous un ingénieur? ». Londres : IMechE. <www.imeche.org>, consulté le 11/07/2016. [IMechE]

Legal Services Consumer Panel. 2013. Breaking the maze. Simplifying legal services regulation. Londres : LSCP. [LSCP]

Plain English Campaign. 2016. «Free Guides ». <www.plainenglish.co.uk/free-guides.html>, consulté le 30/10/2016.

ROSE, Neil. 2011. « Escaping from the regulatory maze ». Legal Futures 19 juillet 2011.

<www.legalfutures.co.uk/blog/escaping-from-the-regulatory-maze>, consulté le 20/05/2016.

ROSE, Neil. 2013. «Call to end 'double regulation' of conveyancers through CQS and lender criteria ». Legal Futures 5 septembre 2013. <www.legalfutures.co.uk/latest-news/call-end-doubleregulation-conveyancers-cqs-lender-criteria>, consulté le 10/07/2016.

Solicitors Regulation Authority. 2011. SRA Handbook. Londres : SRA. <www.sra.org.uk/handbook>, consulté le 7/08/2016. [SRA]

Solicitors Regulation Authority. 2016. « 'Shorter, sharper, clearer' Handbook will benefit consumers and solicitors ». Londres : SRA. <www.sra.org.uk/sra/news/press/handbook-benefitconsumers-solicitors.page>, consulté le 12/07/2016. [SRA]

Spada. 2009. British Professions Today: The state of the sector. Londres : Spada.

\section{Sources secondaires}

BOURDONCLE, Raymond. 1991. « Note de synthèse - La professionnalisation des enseignants : analyses sociologiques anglaises et américaines ». Revue française de pédagogie 94, 73-91.

BOVEnS, Mark, Robert Goodin \& Thomas E. schillemans. 2014. The Oxford Handbook of Public Accountability. Oxford : Oxford University Press.

CHAPOULIE, Jean-Michel. 1973. «Sur l'analyse sociologique des groupes professionnels ». Revue française de sociologie 14/1, 86-114.

DÉTOURBE, Marie-Agnès. 2011. « Contribution à la caractérisation des pratiques d'évaluation dans le domaine de l'enseignement supérieur britannique envisagé comme domaine spécialisé ». Thèse de doctorat, Université Bordeaux 2.

DUFOUR, Françoise. 2011. « Le sigle comme modalité de dissociation énonciative. Le cas des alternances d'usage PMA/Pays les moins avancés ». Mots Les langages du politique 95, 75-87. HUGHES, Everett Cherrington. 1958. Men and their Work. Glencoe : The Free Press.

JEANNOT, Gilles. 1998. Les usagers du service public. Collection Que sais-je ? Paris : Presses universitaires de France.

KOBER-SMITH, Anémone. 2010. « 'We will save the NHS' : la réforme du système de santé anglais sous le New Labour ». Observatoire de la société britannique 8, 69-86.

KOBER-SMITH, Anémone \& Isabelle FERONI. 2005. « La professionnalisation des cadres infirmiers : l'effet de l'action publique en France et en Grande-Bretagne ». Revue française de sociologie 46, 469494.

LAFFONT, Hélène. 2005. « Contribution à la caractérisation de l'anglais pour l'ingénieur ». Thèse de doctorat, Université de Bordeaux 2. 
LE BIANIC, Thomas. 2009. « Les professions face à l'Europe : les psychologues ». In DEMAZIÈRE, D. \& C. Gadéa (dir.), Sociologie des groupes professionnels. Acquis récents et nouveaux défis. Paris : La Découverte, 97-107.

MAROY, Christian. 2007. « Les modes de régulation de l'École ». Revue internationale d'éducation de Sèvres 46, 87-98.

PERCEBOIS, Jacqueline. 2001. «Fonctions et vie des sigles et acronymes en contextes de langues anglaise et française de spécialité » Meta : journal des traducteurs/Meta: Translators' Journal 46/4, 627-645.

PERROUD, Thomas. 2012. «L'indépendance des autorités de régulation au Royaume-Uni ». Revue française d'administration publique 143, 735-746.

PETIT, Michel. 2010. « Le discours spécialisé et le spécialisé du discours : repères pour l'analyse du discours en anglais de spécialité ». E-rea 8/1. <www.erea.revues.org/1400>, consulté le $14 / 06 / 2016$.

RAFFENNE, Coralie. 2010. « La nouvelle gouvernance de la santé au Royaume-Uni ou les apories de la gouvernementalité néolibérale ». Observatoire de la société britannique 8, 271-296.

<www.osb.revues.org/944>, consulté le 2/06/2016.

RESCHE, Catherine. 2013. Economic Terms and Beyond: Capitalising on the Wealth of Notions. How researchers in specialised varieties of English can benefit from focusing on terms. Berne : Peter Lang, coll. Linguistic Insights $n^{\circ} 176$.

RESCHE, Catherine. 2015. « Mapping out research paths to specialised domains and discourse: the example of business cycles and financial crises ». Journal of Teaching English for Academic and Specific Purposes 3/2, 215-228.

\section{NOTES}

1. Voir, par exemple, le rapport «Breaking the maze. Simplifying legal services regulation » publié par le Legal Services Consumer Panel (2013).

2. On peut citer, pour ce qui est de la régulation du milieu de la santé, le rapport «The State of Medical Education and Practice in the UK », publié par le General Medical Council en 2013 et le rapport « Professionalism in Healthcare Professionals » publié par le Health and Care Professions Council en 2011.

3. Voir le code de conduite des avocats «SRA Handbook », publié par la Solicitors Regulation Authority en 2011, ou celui des ingénieurs en génie civil publié par l'Institution of Civil Engineers en 2014.

4. Dans le milieu de la santé, le guide «Good Medical Practice», publié par le General Medical Council depuis 1995, constitue un manuel de référence pour la diffusion des bonnes pratiques médicales.

5. Nous renvoyons le lecteur aux guides « Tomorrow's Doctors» (General Medical Council, 2009) et « Your Career as a Barrister » (The Bar Council 2013).

6. Pour les médecins, cette liste figure à la page suivante du site internet du General Medical Council, l'organisme de régulation des médecins généralistes et spécialistes au Royaume-Uni : <www.gmc-uk.org/education/undergraduate/awarding_bodies.asp>, consulté le 12/06/2016.

7. Pour plus d'informations, voir < www.gmc-uk.org/doctors/registration_applications/ 24986.asp>, consulté le 07/04/2016. 
8. C'est tout particulièrement le cas dans le domaine de la santé, où tout médecin spécialiste (Specialist Doctor) doit impérativement être membre du Royal College de sa spécialité pour pouvoir exercer sa profession. L'organisme délivre au médecin une certification appelée «licence to practise " qui lui permet d'exercer sa spécialité et lui propose également des activités de formation continue. Dans le domaine du droit, en revanche, les Solicitors et les Barristers sont tenus d'adhérer respectivement à la Law Society et au Bar Council seulement au stade des études, en tant que membres étudiants. À la suite de l'obtention de leur diplôme, les Solicitors ne sont pas obligés de renouveler leur inscription à l'association professionnelle, mais ils restent soumis aux règles de déontologie et de discipline qu'elle édicte. Enfin, pour les ingénieurs, l'adhésion à une association importante comme la Royal Academy of Engineering (RAEng), dont les membres sont des personnalités reconnues du monde scientifique, n'est pas non plus obligatoire pour exercer la profession mais demeure garante d'un certain prestige (Laffont $2005: 148$ ).

9. Recherche menée sur le site généraliste d'offres d'emploi Indeed, le 18 juin 2016, concernant les postes de « Chemical Engineer », « Building Services Engineer » et « Water Engineer ».

10. Ces organismes font partie de l'Administration centrale mais ne sont pas dirigés par un ministre. Ils échappent ainsi au principe de responsabilité ministérielle (ministerial responsibility) et à l'influence politique (Perroud 2012 : 741).

11. La Professional Standards Authority, organisme chargé de veiller à la mise en œuvre et au respect des politiques de régulation dans le domaine de la santé, publie ainsi des informations concernant les neuf principaux organismes de régulation sur son site internet <http:// www.professionalstandards.org.uk/ what-we-do/our-work-with-regulators/find-a-regulator>.

12. Les abréviations utilisées pour désigner le diplôme de master en médecine diffèrent ainsi suivant les universités. On trouve par exemple l'abréviation $M B$ ChB dans les universités écossaises et dans certaines universités anglaises (Birmingham, Bristol, Leicester, Liverpool, Manchester, Sheffield, Warwick). D'autres universités (University of East Anglia, University of London et University of Newcastle ainsi que la Hull York Medical School) remettent un MBBS à leurs étudiants. Dans les universités de Cardiff et de Galles, le diplôme reçoit l'abréviation BM BCh. L'université de Nottingham, la Peninsula Medical School et la Brighton Sussex Medical School utilisent BM BS. Enfin, l'université de Southampton remet des BM et l'université de Cambridge des MB BChir.

13. Nous renvoyons le lecteur à la page suivante du site du Health and Care Professions Council (HCPC) : <www.hpc-uk.org/aboutus/aimsandvision/principles/>, consultée le 03/06/2016.

14. Ce sigle est issu de la dénomination latine «Medicinae Baccalaureus Chirurgiae Baccalaureus ».

15. C'est ce que précise Hélène Laffont à propos de certaines associations professionnelles d'ingénieurs comme l'Energy Institute, issu du regroupement, en 2003, de l'Institute of Petroleum avec l'Institute of Energy (2005: 146).

16. Les organismes de régulation du domaine de la santé sont disponibles à la page suivante : <www.osteopathy.org.uk/about-us/uk-health-regulators/>; ceux du domaine du droit sont répertoriés ici : <www.legalservicesboard.org.uk/can_we_help/approved_regulators/>.

17. La Plain English Campaign est une association qui, depuis sa création en 1979, mène plusieurs initiatives pour simplifier la langue anglaise administrative, juridique, commerciale et financière, en mettant l'accent sur un style de communication clair et efficace. Elle propose notamment des guides pratiques d'aide à la rédaction, des glossaires détaillés et des formations pour aider les professionnels à rendre plus accessible le contenu de leurs écrits ou de leurs propos. Dans les échanges entre professionnels et usagers, les efforts de rédaction ou de formulation réalisés doivent permettre une meilleure compréhension entre acteurs. 


\section{RÉSUMÉS}

Cet article s'intéresse à la fonction de régulation de trois milieux professionnels au RoyaumeUni : la santé, le droit et l'ingénierie. L'accès à ces trois milieux est fortement régulé : il est conditionné par de nombreuses exigences visant à garantir la compétence et le professionnalisme des praticiens auprès du public. Notre étude s'appuie sur un corpus réunissant différents textes de régulation (rapports d'activité des régulateurs et des associations d'usagers, recommandations de bonnes pratiques, codes de conduite, guides d'information), publiés entre 1995 et 2016. Notre corpus présente une grande diversité terminologique, qui se manifeste dans la diversité des appellations et des statuts conférés aux régulateurs, dans les emprunts à d'autres domaines d'application, et dans la prolifération des sigles et acronymes. Cette diversité montre que l'organisation des milieux professionnels s'est complexifiée depuis les années 1990. L'analyse des pratiques et des discours de régulation vise à apporter une meilleure compréhension des milieux professionnels de la santé, du droit et de l'ingénierie.

This article focuses on the regulatory function that characterises the health, law and engineering professions in the United Kingdom. Access to these professions is highly regulated: it is determined by numerous requirements, which are assumed to guarantee the competence of practitioners. Our study relies upon a corpus gathering different regulatory discourses - reports by regulators and users' associations, recommendations of good practice, codes of conduct, information guides - published between 1995 and 2016. Our corpus shows a great terminological diversity, which can be seen in the names and statuses given to regulators, in the words borrowed from other domains of application, and in the profusion of abbreviations and acronyms. This diversity shows that the organisation of professions has become more complex since the 1990s. The study of the regulatory practices and discourses aims to improve the general understanding of the health, law and engineering professions.

\section{INDEX}

Keywords : profession, regulatory discourse, regulatory function, specialised domain Mots-clés : discours de régulation, domaine spécialisé, fonction de régulation, milieu professionnel

\section{AUTEUR}

\section{CAROLINE BENEDETTO}

Caroline Benedetto a enseigné l'anglais à l'École nationale de la statistique et de l'administration économique ParisTech (ENSAE ParisTech) et à l'Institut d'études politiques de Paris (Sciences Po). Elle prépare une thèse de doctorat à l'Université de Bordeaux sous la direction de Michel Petit. Ses recherches se situent dans le champ de l'anglais de spécialité et portent plus particulièrement sur les discours de régulation en lien avec les professions au Royaume-Uni. caroline.benedetto@sciencespo.fr 